\title{
Structural analysis of mycobacterial homoserine transacetylases central to methionine biosynthesis reveals druggable active site
}

\begin{abstract}
Catherine T. Chaton ${ }^{1}$, Emily S. Rodriguez ${ }^{1,2}$, Robert W. Reed ${ }^{1,3}$, Jian $\mathrm{Li}^{1,4}$, Cameron W. Kenner ${ }^{1,5}$ \& Konstantin V. Korotkov ${ }^{1 *}$

Mycobacterium tuberculosis is the cause of the world's most deadly infectious disease. Efforts are underway to target the methionine biosynthesis pathway, as it is not part of the host metabolism. The homoserine transacetylase MetX converts L-homoserine to $O$-acetyl-L-homoserine at the committed step of this pathway. In order to facilitate structure-based drug design, we determined the high-resolution crystal structures of three MetX proteins, including M. tuberculosis (MtMetX), Mycolicibacterium abscessus (MaMetX), and Mycolicibacterium hassiacum (MhMetX). A comparison of homoserine transacetylases from other bacterial and fungal species reveals a high degree of structural conservation amongst the enzymes. Utilizing homologous structures with bound cofactors, we analyzed the potential ligandability of MetX. The deep active-site tunnel surrounding the catalytic serine yielded many consensus clusters during mapping, suggesting that MtMetX is highly druggable.
\end{abstract}

Mycobacterium tuberculosis (Mtb) is the causative agent of tuberculosis (TB), a persistent global health threat. In 2017, TB was responsible for the deaths of 1.3 million HIV-negative people and an additional 300,000 deaths among the HIV-positive population ${ }^{1,2}$. Approximately 10 million people contracted TB that same year ${ }^{2}$. Despite increased focus since WHO declared TB as a global health emergency back in 1993, progress towards fighting it has been mixed. It ranks among the top ten causes of death among the world's population and is the single most deadly infectious disease, claiming more lives than HIV/AIDS ${ }^{2}$. Survivors of TB infections can also suffer significantly reduced quality of life ${ }^{3}$.

The bacilli Calmette-Guerin (BCG) vaccine is capable of protecting children from the most severe forms of $\mathrm{TB}$ and is still the preferred method for disease control. However, no vaccine is capable of preventing TB infection in adults either pre or post-exposure ${ }^{4}$. Existing anti-TB therapy requires treatment of six months or more with a combination of multiple therapeutic agents: rifampicin, ethambutol, isoniazid, and pyrazinamide. Treatment with first-line drugs leads to a cure rate of about $85 \%$ of those with drug-susceptible TB strains ${ }^{2}$. The side effects and length of these treatments has led to compliance issues. This fact, combined with the lack of care in some areas of the world, has spurred the development of multi-drug resistant TB (MDR-TB); 6\% of these MDR-TB cases are so antibiotically hardened that they are classified as extensively drug-resistant TB (XDR-TB) ${ }^{2}$. MDR-TB and XDR-TB infections have limited treatment options which are not always successful, particularly in the case of immunocompromised patients and XDR-TB. Current therapeutic regimens are often toxic, require long durations of treatment time, and are estimated to increase treatment costs 8 to 15 -fold compared to treating regular TB infections ${ }^{5}$. This difference is even more extreme in the case of XDR-TB with costs being 25 to 32 -times higher. There is a clear need for practical, low-cost TB therapies with orthogonal activity to current antibiotic options.

MetX (previously mis-annotated as MetA in the reference genome of M. tuberculosis) is an L-homoserine $O$-acetyltransferase, commonly also referred to as a homoserine transacetylase (HTA) and is a crucial enzyme in the

\footnotetext{
${ }^{1}$ Department of Molecular \& Cellular Biochemistry and the Center for Structural Biology, University of Kentucky, Lexington, KY, 40536, USA. Present address: Department of Chemistry \& Biochemistry, Ohio State University, Columbus, $\mathrm{OH}, 43210, \mathrm{USA}$. ${ }^{3}$ Present address: Division of Regulatory Services, College of Agriculture, Food and Environment, University of Kentucky, Lexington, KY, 40546, USA. 'Present address: Gannan Medical University, Ganzhou, Jiangxi, 341000, China. ${ }^{5}$ Present address: Georgetown College, Georgetown, KY, 40324, USA. *email: kkorotkov@uky.edu
} 


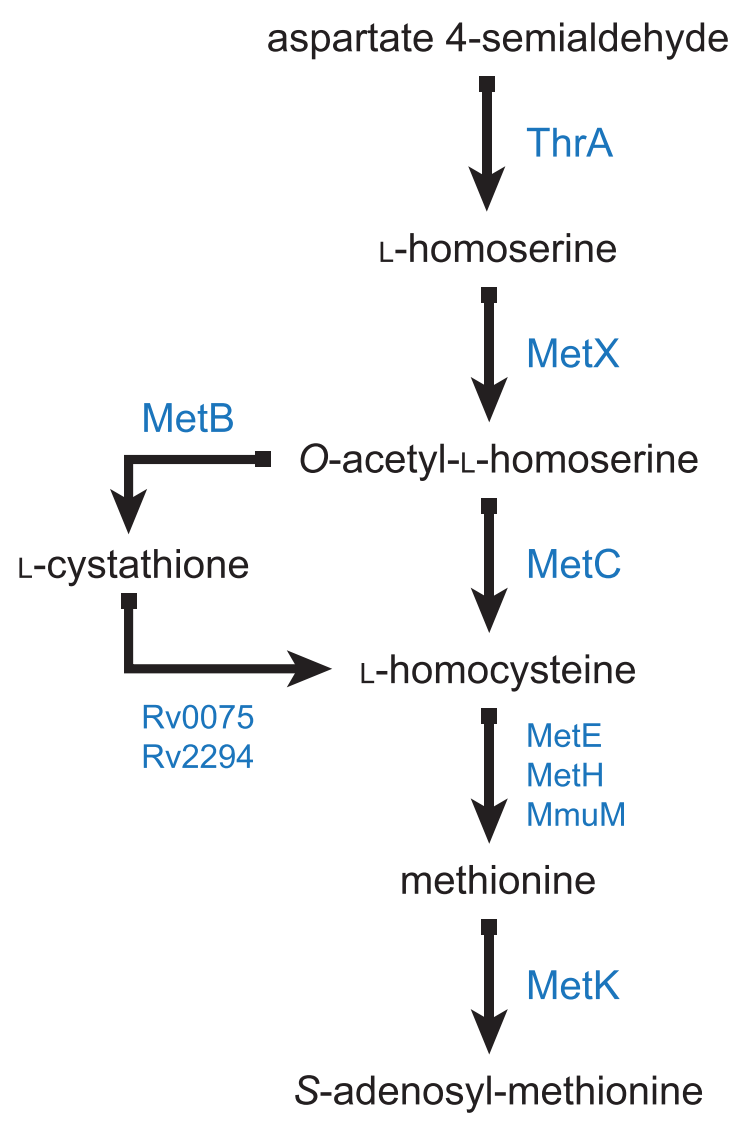

Figure 1. Overview of the M. tuberculosis biosynthesis pathways dependent on MetX converting L-homoserine into $\mathrm{O}$-acetyl-homoserine. Inhibition of MetX will affect not only methionine synthesis but also the production of SAM and threonine, among other necessary metabolites.

biosynthesis of methionine and threonine (Fig. 1$)^{6}$. It catalyzes the conversion of L-homoserine to $O$-acetyl-L-homoserine (OAHS) by transfer of an acetyl group from Acetyl-CoA to the $\gamma$-hydroxyl of homoserine ${ }^{7}$. OAHS is an essential precursor to methionine as well as other bacterial metabolites, such as $S$-Adenosyl-L-methionine (SAM). The modification of $\mathrm{L}$-homoserine is a committed step in methionine and SAM synthesis.

Bacterial studies have shown that deletion of met2 gene, which encodes an analogous HTA, is lethal without methionine supplementation ${ }^{8,9}$. Further studies using immunocompetent and immunocompromised mice demonstrate that deletion of $m e t X$ generates auxotrophic mutants unable to establish infection ${ }^{10}$. If starved of threonine and methionine in vitro, Mtb $\Delta$ met $X$ dies quickly. Furthermore, $\Delta$ met $X$ mutant strain is unable to proliferate inside of human macrophages. Also, it has recently been demonstrated that met $X$ is required for maintaining bacterial survival during chronic Mtb infection ${ }^{11}$. Together, these data suggest that Mtb is unable to scavenge biosynthetic intermediates from the host for methionine synthesis, making for a uniquely exploitable vulnerability for the development of antibacterial agents ${ }^{12}$.

Efforts are already underway to inhibit HTA in Cryptococcus neoformans for use as an antifungal agent ${ }^{8}$. Targeting of a similar pathway for aspartate production has already yielded some promising selective inhibitors for Streptococcus pneumoniae and Vibrio cholerae ${ }^{13}$. A similar structurally guided approach to discovering specific inhibitors is an attractive alternative to traditional antibiotic killing of MDR-TB and XDR-TB.

Here we report the structures of three homologous MetX enzymes from Mycobacterium tuberculosis (MtMetX), Mycolicibacterium abscessus (MaMetX), and Mycolicibacterium hassiacum (MhMetX) and compare them to previously solved structures of HTAs in order to begin development of selective inhibitors of $M t$ MetX via a structure-based approach. Using the $M t$ MetX structure as a guide, we also elucidate the druggability of the enzyme and propose that it is an excellent candidate for small molecule drug discovery.

\section{Results}

Overview of mycobacterial MetX structures. The three solved MetX structures include residues 15-70, 77-372 from MhMetX (Fig. 2A), 10-379 from MaMetX (Fig. 2B), and 7-372 from MtMetX (Fig. 2C). Two copies of each monomer exist in the asymmetric unit of all three structures. MetX can be divided into two distinct structural domains, the catalytic domain, and the lid domain.

The organization of the catalytic domains' fold marks MetX as members of the $\alpha / \beta$-hydrolase super-family. It is a highly diverse family that includes proteases, lipases, and esterases, among many others ${ }^{14-16}$. A canonical 8 -stranded $\beta$-sheet fold with twisted, parallel topology forms the core of $\alpha / \beta$-hydrolases ${ }^{17}$. Several $\alpha$-helices flank 

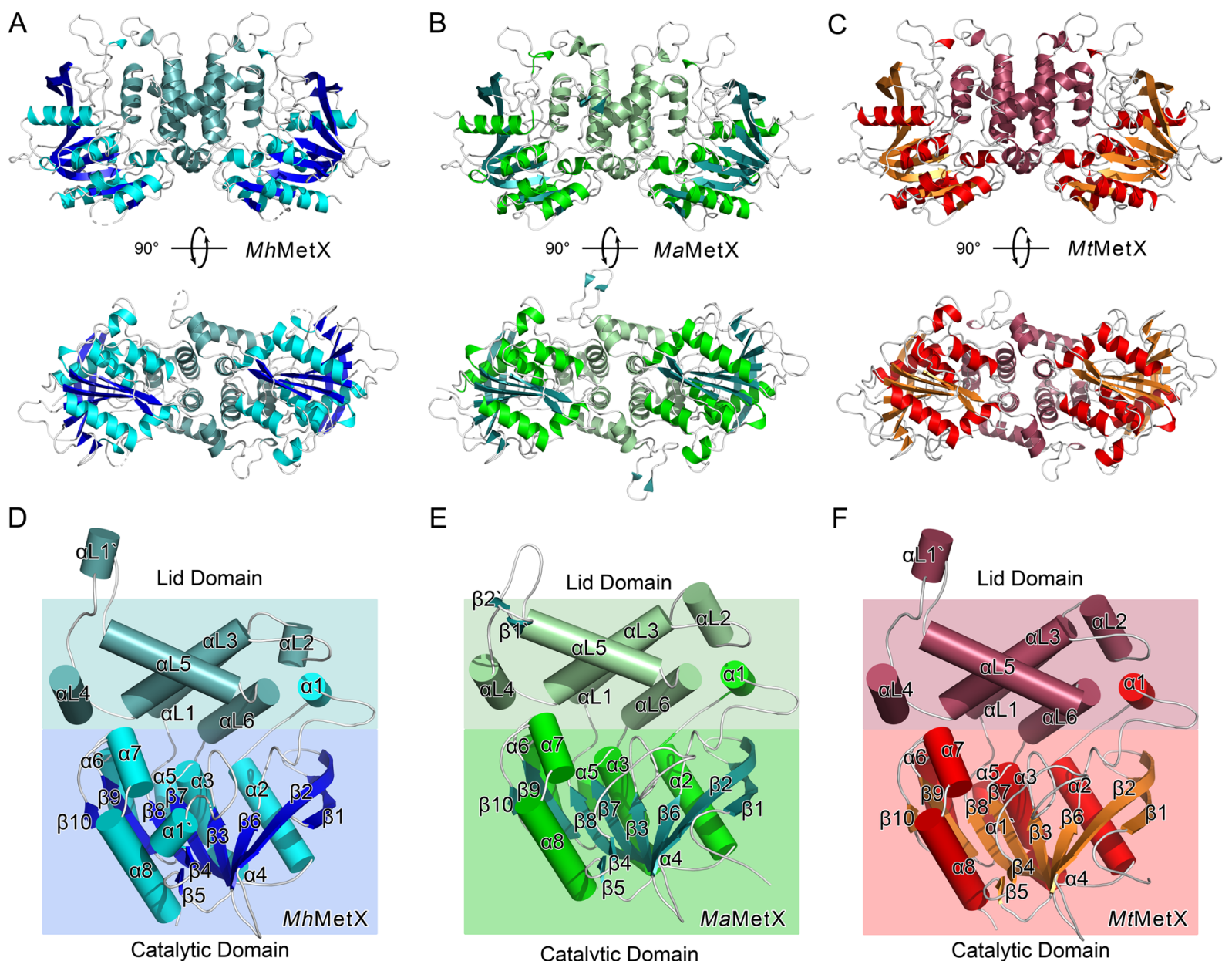

$\mathrm{E}$

F

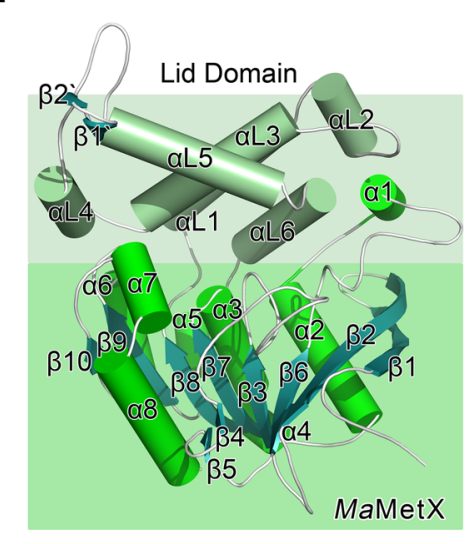

Catalytic Domain

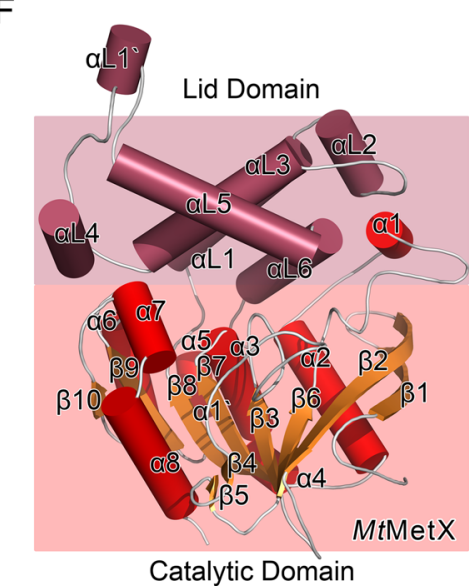

Figure 2. (A) Ribbon diagram of $M h M e t X$. Catalytic domain sheets are shown in blue, helices of the catalytic domain are in cyan, and the lid domain secondary structure is shown in blue-green. (B) Ribbon diagram of MaMetX. Catalytic domain sheets are shown in green-blue, helices in bright green, and the lid domain in mint. (C) Ribbon diagram of MtMetX. Catalytic domain sheets are shown in orange, helices in red, and the lid domain in burgundy. (D-F) Simplified cartoon structures of MhMetX, MaMetX, and MtMetX monomers with topological labels and both domains.

either face of this fold, though their number and location are different depending on the specific protein. The catalytic domain comprises residues 15-181, 297-372 of MhMetX (Fig. 2D), residues 17-183, 304-379 of MaMetX (Fig. 2E) and residues 17-181, 311-372 of MtMetX (Fig. 2F). The catalytic domain contains the active site tunnel with its a canonical catalytic triad.

Assembly occurs at an anti-parallel four-helix bundle motif $(\alpha \mathrm{L} 1$ and $\alpha \mathrm{L} 3)$ in the lid domain. The total interface area of the dimer is $\sim 1700 \AA^{2}{ }^{18}$. Two additional helices help to strengthen the interaction with hydrogen bonds and van der Waals contacts ( $\alpha \mathrm{L} 4$ and $\alpha \mathrm{L} 5)$. Like other previously studied HTAs, MetX forms solution dimers at this interface. These dimers have been shown to be physiologically relevant in other HTAs and are likely important for MetX as well ${ }^{19}$. The lid domain comprises residues 184-285 of both MhMetX and MtMetX, and residues 187-292 of MaMetX, between $\beta 8$ and $\alpha 5$ (Fig. 3A).

The space between the catalytic and lid domains forms a deep active-site tunnel. At the end of this tunnel sits the nucleophilic serine residue. The tunnel is lined with polar residues highly conserved among other known HTA structures. Thr61/61/64, Arg227/227/230, Tyr234/234/237, and Asp351/351/358 for MhMetX, MtMetX, and $\mathrm{MaMetX}$ respectively all surround the active site to help facilitate the binding of acetyl-CoA and homoserine (Fig. 3B $)^{20}$.

Catalytic mechanism. The catalytic triad of Nucleophile-His-acid is the $\alpha / \beta$-hydrolase family's most conserved feature. Just as in other known HTA structures, $M t \mathrm{HTA}, M h \mathrm{HTA}$, and MaHTA contain a serine, aspartic acid, and histidine in the active site. HTAs have a serine between $\beta 7$ and $\alpha 3$, an aspartic acid on the loop between $\beta 9$ and $\alpha 6$, and histidine on $\alpha 7$ for these residues. For MtHTA and MhHTA, Ser157, Asp320, and His350 comprise the active site; MaHTA's triad is comprised of Ser160, Asp327, His357 (Fig. 4A). The catalytic serine sits at the end of a deep catalytic tunnel (Fig. 4B).

Studies in H. influenzae and Schizosaccharomyces pombe suggest that the mechanism of HTAs is based on a "ping-pong" reaction ${ }^{21}$. In the proposed mechanism, acetate is first transferred to serine from acetyl-CoA in the 


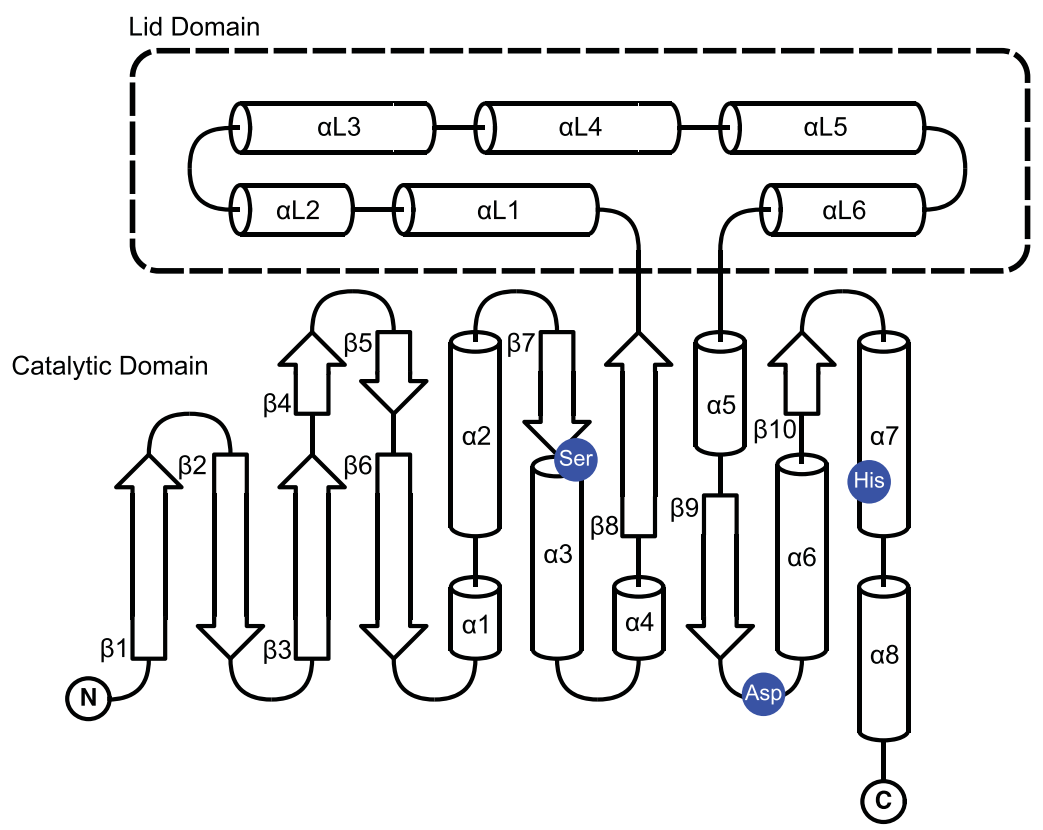

B

M. tuberculosis
M. tuberculosis
M. hassiacum
M. abscessus
M. smegmatis
H. influenzae
S. aureus
L. interrogans
M. tuberculosis
M. tuberculosis
M. hassiacum
M. abscessus
M. smegmatis
H. influenzae
S. aureus
L. interrogans
M. tuberculosis
M. tuberculosis
M. hassiacum
M. abscessus
M. smegmatis
H. influenzae
S. aureus
L. interrogans
M. tuberculosis
M. tuberculosis
M. hassiacum
M. abscessus
M. smegmatis
H. influenzae
S. aureus
L. interrogans
M. tuberculosis
M. tuberculosis
M. hassiacum
M. abscessus
H. smegmatis
H. influenzae
S. aureus
L. interrogans

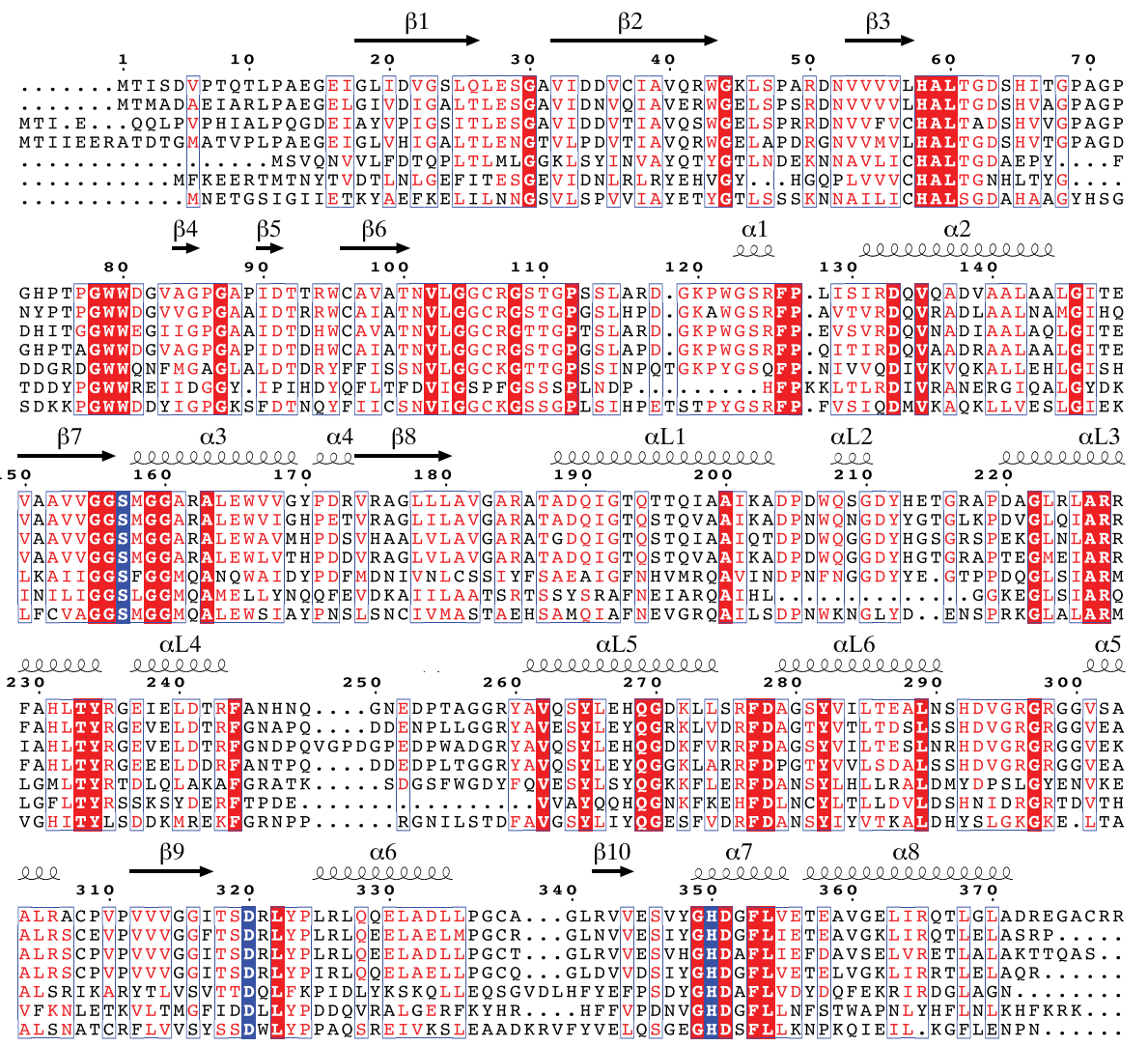

Figure 3. (A) Simplified schematic diagram of conserved secondary structural elements of MetX. (B) Sequence alignments of $M h M e t X, M a M e t X, M t M e t X, H i H T A, S a H T A$, and $L i H T A$ with assigned secondary structure denoted above. Catalytic residues are highlighted in blue.

"ping" step to create an acetyl-enzyme intermediate through an attack of the acetyl-CoA thioester bond. In the "pong," L-homoserine breaks down a tetrahedral intermediate of the acetyl-enzyme to complete the transfer to $\mathrm{OAH}^{20}$. Histidine and aspartic acid function as bases to activate the serine residue for attack and can also most likely assist in deprotonation of the $\mathrm{L}$-homoserine ${ }^{21}$.

The active site residues of $M t \mathrm{MetX}, M h \mathrm{MetX}, M a \mathrm{MetX}$, and $M s \mathrm{HTA}$ have similar geometry to other bacterial and fungal HTAs. Just as in other structures, the serine sits in a strained conformation at the end of the active site tunnel, ideally positioned for its function as the nucleophile. The histidine and aspartic acid residues are within 
A

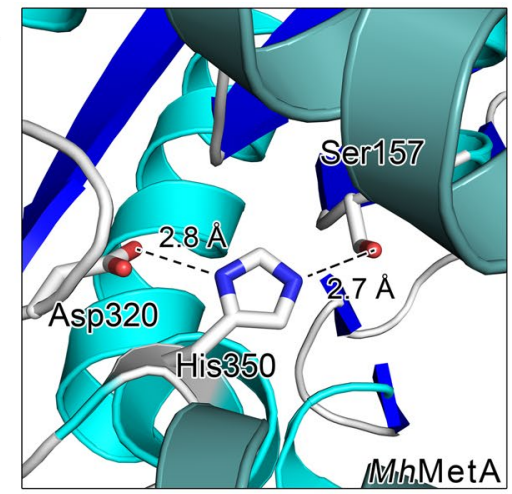

$\mathrm{B}$

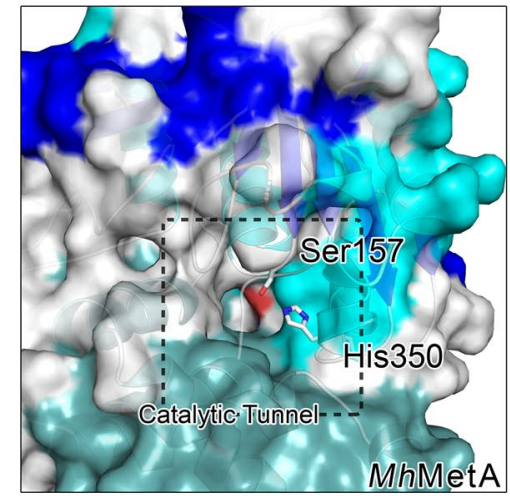

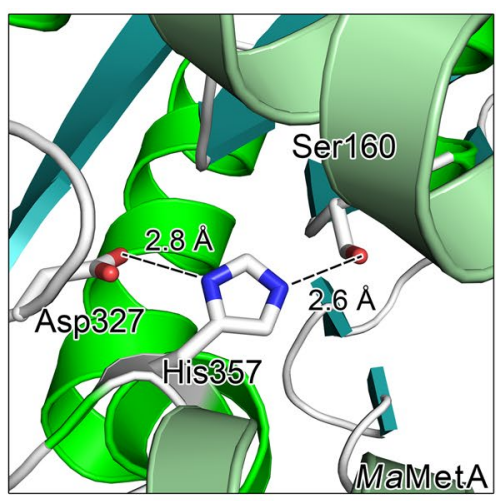
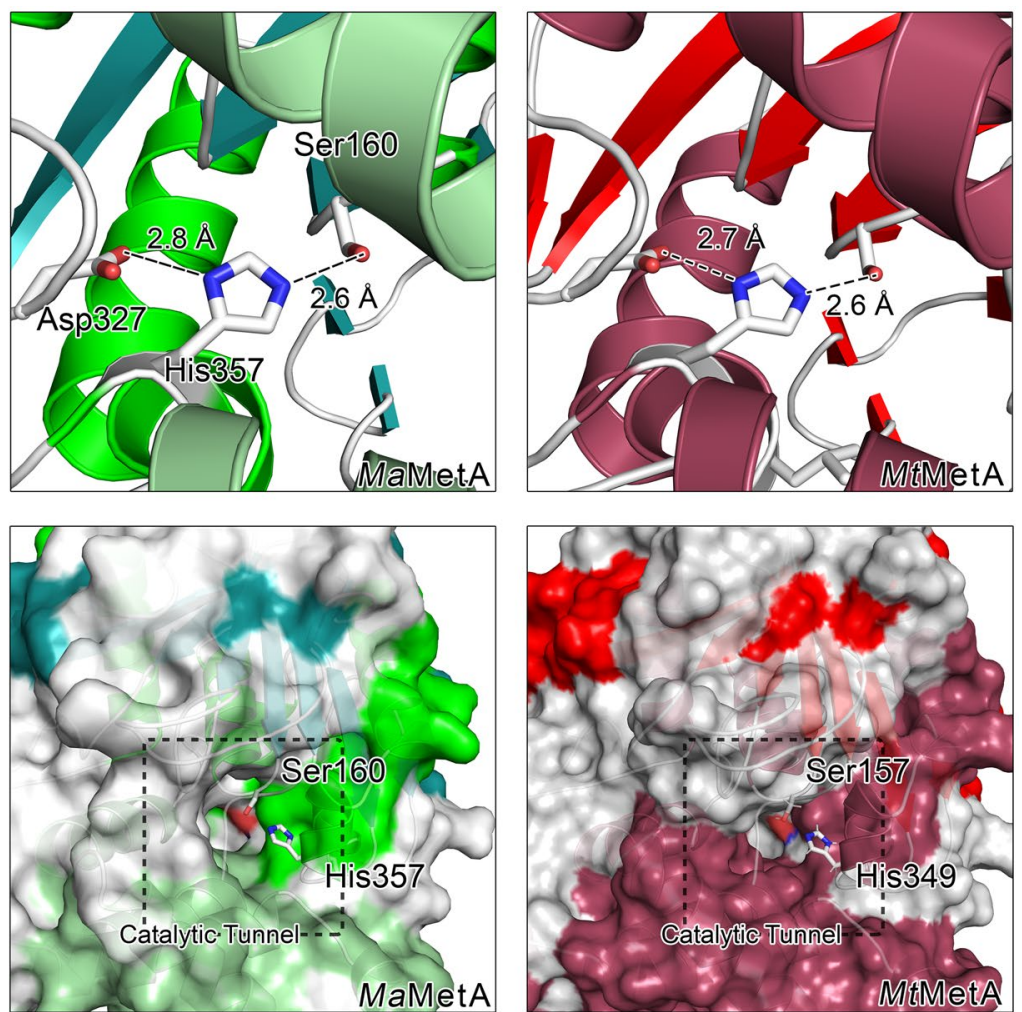

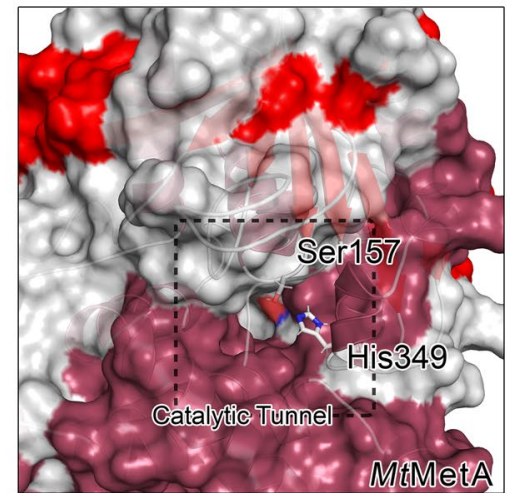

Figure 4. (A) Ribbon diagrams of $M h \mathrm{MetX}, \mathrm{MaMetX}$, and MtMetX active site residues. Catalytic residues are shown in stick representation with key hydrogen bonding distances between heavy atoms. (B) Cross-sectional diagrams of $M h \mathrm{MetX}, \mathrm{MaMetX}$, and MtMetX catalytic tunnels with the catalytic serine and histidine residues highlighted.

hydrogen-bonding distance for activation of the serine (Fig. 4). The active sites of LiHTA and SaHTA show the most deviation. In the structure, His344 of LiHTA exists in two different conformations with equivalent occupancy $^{22}$. In the $S a H T A$ structure, His 296 is more disordered with a high B-factor $\left(B=72 \AA^{2}\right)$ for its imidazole ring $^{23}$. Temperature factors of the equivalent rings in $M h \mathrm{MetX}$ is $\mathrm{B}=19 \AA^{2}$, while both $M t \mathrm{MetX}$ and $M a \mathrm{MetX}$ are $\mathrm{B}=21 \AA^{2}$, values very similar to the HiHTA structure with $\mathrm{B}=16 \AA^{2}$.

Structural comparisons to other HTAs. The overall fold is similar to previously solved HTAs, specifically $M s$ HTA (PDB entry 6IOG) ${ }^{24}$, HiHTA (PDB entry 2B61) ${ }^{25}$, SaHTA (PDB entry 4QLO) ${ }^{23}$, LiHTA (PDB entry $2 \mathrm{PL5})^{22}$. The three solved MetXs are most similar in length to MsHTA (374 residues), with $L i$ HTA (366 residues) and $\mathrm{SaHTA}$ (322 residues) being smaller proteins than MetX and HiHTA (377 residues) being slightly longer. The significant differences lie in the loop lengths and a few short secondary structural elements. The most notable secondary structure difference occurs in the length of $\beta 1$ and $\beta 2$, which are both extended in the $M t$ MetX, MhMetX, MaMetX, and MsHTA structures when compared to the other related HTA structures. In LiHTA, HiHTA, and $\mathrm{SaHTA}$, these sheets are both subdivided by loops, whereas they are continuous in MtMetX, MhMetX, MaMetX, and $M s$ HTA. The extended loop between $\beta 3$ and $\beta 4$ show differences in secondary structure content, length, and orientation. Both $M h \mathrm{MetX}$ and $\mathrm{HiHTA}$ contain a short sequence with helical propensity adjacent to $\beta 4$. MaHTA and $L i$ HTA have no secondary structure as assigned by DSSP in this same region ${ }^{26,27}$, while SaHTA is unique in substituting $\beta 4$ and $\beta 5$ for a longer helix.

As the lid domains are the least conserved among the six HTA structures and within the $\alpha / \beta$-hydrolase family as a whole, it is not surprising that this region has some of the most substantial structural deviations. The loop between $\alpha \mathrm{L} 4$ and $\alpha \mathrm{L} 5$ appears unique in each structure. MtMetX and MsHTA both contain a small helical region $\left(\alpha 1^{\prime}\right)$; MaHTA features a unique pair of short anti-parallel sheets $\left(\beta 1^{\prime}, \beta 2^{\prime}\right)$. HiHTA merely contains an unstructured loop, while $\mathrm{SaHTA}$ omits the majority of the residues entirely, with only a short linker between $\alpha \mathrm{L} 4$ and $\alpha$ L5. $L i$ HTA is perhaps the most variant, as its loop affects the length and orientation of $\alpha$ L5. Due to the high degree of variability, this loop is likely not critical for MetX's function or assembly.

The polar residues which line the active site tunnel show conservation between variants of HTA. Additionally, the motifs surrounding each are nearly identical. Notably, Thr61 (substituted for Ser in LiHTA) is in the middle of a HALTGD motif, and Asp351 sits next to the conserved region, adjacent to the catalytic His, in a GHD(G/A) FL motif. While the lid domain contains a much lower amount of structural conservation globally, the stretch of highest convergence appears in $\alpha \mathrm{L} 3$, which contains both Arg227 and Tyr234 and directly forms the other side of the catalytic tunnel. $\alpha \mathrm{L} 1$, the other partner in the four-helix bundle with $\alpha \mathrm{L} 3$, also shows a fair degree of sequence 


\begin{tabular}{|c|c|c|c|c|c|c|c|c|}
\hline & & MhMetX & MaMetX & MtMetX & MsHTA & HiHTA & SaHTA & LiHTA \\
\hline \multirow{3}{*}{ MhMetX (5W8O) } & $s^{\mathrm{a}}$ & - & 970 & 972 & 971 & 906 & 693 & 806 \\
\hline & RMSD & & 0.70 & 0.59 & 0.52 & 1.50 & 2.39 & 2.82 \\
\hline & similarity $^{\mathrm{b}}$ & & $79.56 \%$ & $86.59 \%$ & $84.36 \%$ & $52.79 \%$ & $41.62 \%$ & $56.39 \%$ \\
\hline \multirow{3}{*}{ MaMetX (5W8P) } & $s$ & 970 & - & 1074 & 1072 & 903 & 680 & 817 \\
\hline & RMSD & 0.70 & & 0.58 & \begin{tabular}{|l|l|}
0.68 \\
\end{tabular} & 1.99 & 2.57 & 3.01 \\
\hline & similarity & $79.56 \%$ & & $83.74 \%$ & $82.16 \%$ & $54.22 \%$ & $38.29 \%$ & $53.66 \%$ \\
\hline \multirow{3}{*}{ MtMetX (6PUX) } & $s$ & 972 & 1074 & - & 1077 & 904 & 670 & 813 \\
\hline & RMSD & 0.59 & 0.58 & & 0.53 & 1.94 & 2.55 & 3.04 \\
\hline & \begin{tabular}{|l|} 
similarity \\
\end{tabular} & $86.59 \%$ & $83.74 \%$ & & $88.49 \%$ & $51.52 \%$ & $39.29 \%$ & $54.79 \%$ \\
\hline
\end{tabular}

Table 1. FATCAT pairwise flexible alignment of MetX and HTA structures. ${ }^{a}$ Raw structural similarity scores (s) shown as all $P$-values rounded to zero. Structures with a probability value of $P<0.05$ are considered

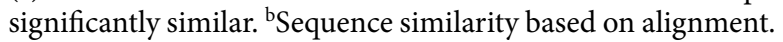

conservation. Interestingly, SaHTA stands as an outlier when comparing tunnel dimensions, being much narrower and more restricted when compared to the other four HTA structures.

Alignment of MtMetX, MaMetX, and MhHTA structures using the FATCAT algorithm ${ }^{3}$ demonstrate their high degree of structural similarity (Table 1). Across the examined monomers, all are significantly similar to one another $(P<0.05)$. Structural alignment RMSDs ranged from $0.52-3.04$, and sequence similarity was in the range of 38.2-88.5\%. MhMetX, MaMetX, and MtMetX ranked in the list of closest structural neighbors currently available on the PDB when applying FATCAT to all related structures. Interestingly, MsHTA is as good if not better an approximation to $M t$ MetX as either MhMetX or MaMetX, suggesting that it may function as a good analog in assays where $M t$ MetX cannot be directly utilized. However, when assaying $M t$ MetX to determine its viability for crystallography, the $\mathrm{T}_{\mathrm{m}}$ was found to be between $40-41^{\circ} \mathrm{C}$ by differential scanning fluorimetry (DSF) between $\mathrm{pH}$ 6.9-8.5 in Tris-HCl. Because of MtMetX's relative thermal stability under physiological conditions, we have chosen to focus on $M t$ MetX when investigating future drug development.

Potential ligandability of MetX. Druggability of a protein is understood to be a measure of the relative ease of developing a small molecule, which will effectively modulate its activity in vivo ${ }^{28,29}$. Druggability depends on the pharmacodynamics and pharmacokinetics of the host and the pathogen making it difficult to predict computationally. Ligandability is a necessary condition for druggability, but is a more easily quantified metric for the development of inhibitors ${ }^{30}$. Understanding the ligandability is a critical first step before embarking on drug discovery. An estimated $60 \%$ of small-molecule searches fail due to the target site not being sufficiently druggable, which is positively correlated with not being sufficiently ligandable $\mathrm{e}^{31,32}$. The availability of high-resolution structures for MetX opens up the possibility of performing direct therapeutic target discovery, provided it proves to be sufficiently ligandable.

The fundamental principle of drug discovery is that biologically active ligands are complimentary in molecular features and shape to the receptor. These can include physiochemical properties such as hydrophobicity, size, as well as the enclosure of the binding pocket and its promiscuity ${ }^{33,34}$. A strictly predominantly hydrophobic pocket might indicate a promiscuous binding site that may accommodate a wide-varying of ligands in different modes, some hydrophobic patches are still nevertheless ideal for ligand design. All three solved MetX have a similar, conserved hydrophobic patch (Fig. 5A-C) running along the inside of the active site tunnel. Binding models for homoserine (Fig. 5D) and acetyl-CoA (Fig. 5E) were created by hybridizing the MtMetX structures with available $M s$ HTA structures that have been co-crystallized with both substrates ${ }^{24}$ in order to better understand their coordination within the binding pocket. While the hydrophobic patch does not appear critical to the recognition of either, its proximity to the catalytic site residues make it an ideal feature to leverage for designing hydrophobic ringed small molecules with flexible tail groups that could orient inside of the cleft similar to acetyl-CoA.

In order to further understand the active site's ligandability, FTMap was utilized ${ }^{35-37}$. The FTMap algorithm uses a set of small organic probes to sample a protein surface for binding hotspots computationally. Each organic probe is first rigidly docked before being energy minimized. Areas on the protein's surface where multiple probes bind are clusters. Binning these clusters based on their member's average free energy yields a consensus site (CS), a location on the protein's surface where small molecules are likely to bind. A CS strength (S) is defined as the number of probe clusters within the consensus cluster. A cluster of $\mathrm{S}>16$ represents a site targetable by a ligand ${ }^{36,37}$. A second CS should be located somewhere within $8 \AA$ of the primary cluster. Of the eleven CS identified by FTMap, eight reside somewhere within the active site tunnel (Fig. 6). The closest CS near the catalytic Ser have $S=19, S=16$ and $S=13$. One $C S$ with $S=13$ forms across from the active site on the lid domain, but no other high strength CS exists near it, making it an unlikely site for drug binding. MaMetX shows a similar pattern with the top clusters appearing nearly overtop those of $M t$ MetX with $S=26, S=13$ and $S=10$. These results, when overlaid with the hybrid substrate-binding models, suggest that MetX is ligandable.

\section{Discussion}

All three MetX structures show a high degree of overall similarity to previously studied HTAs from both bacteria and fungi. Differences in enzyme size are accounted for by the length of loop regions. Differences in secondary structural elements arise primarily from variations within these loops. All three solved structures crystallized as dimers at the expected interface and orientation that corresponds to predicted physiologically active assembly. 
A

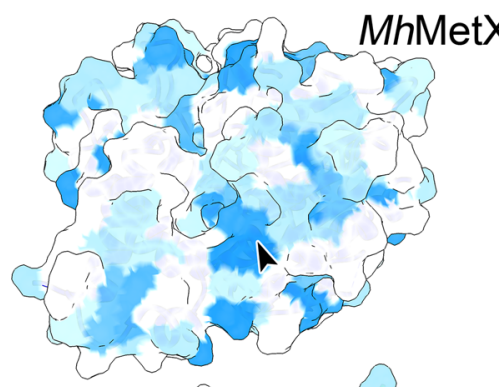

B
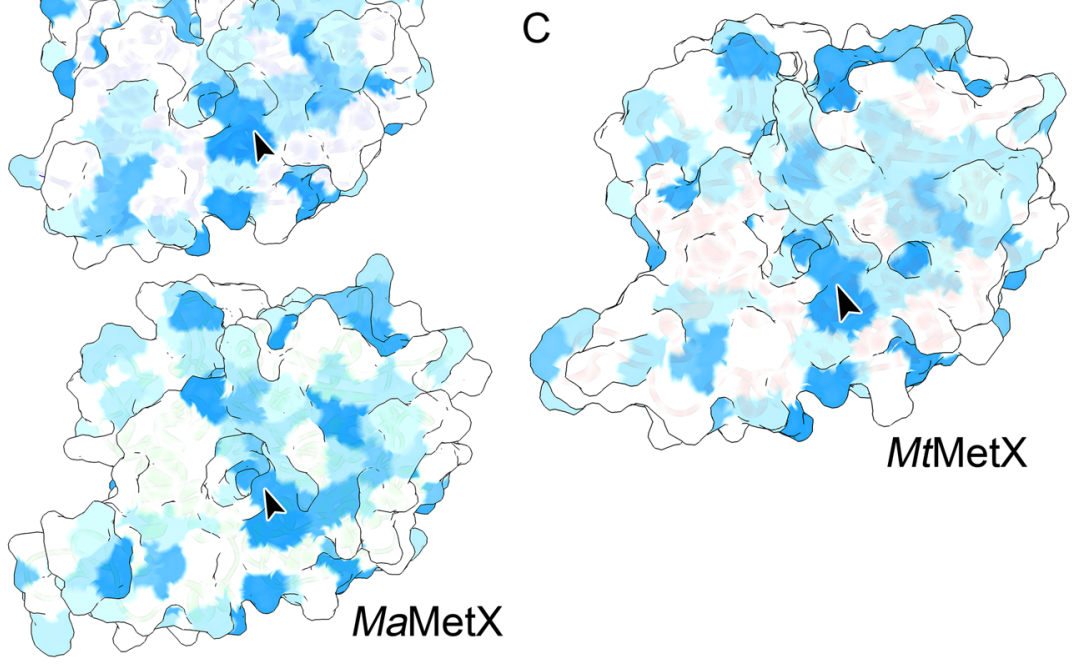

$\mathrm{D}$

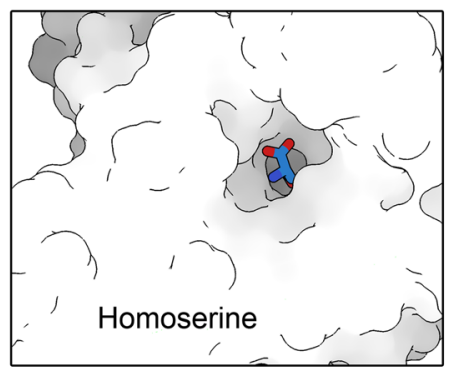

$\mathrm{E}$

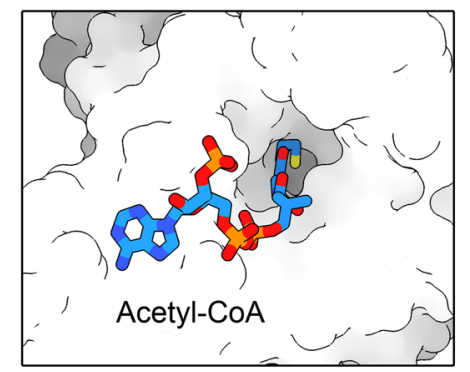

Figure 5. (A-C) Hydrophobicity of surface residues on the Kyte and Doolittle scale of MhMetX, MaMetX, and $M t$ MetX (blue hydrophobic; white hydrophilic). A hydrophobic patch extends from the apex of the active site pocket to the surface of the protein (arrow), which may be an exploitable feature when designing small molecule inhibitors. (D) L-homoserine binding hybrid binding model created using a previously solved $M s \mathrm{HTA} \bullet \mathrm{HSE}$ structure (PDB ID 6IOH) by aligning the monomer backbone with MtMetX followed by energy minimization. (E) Acetyl-CoA binding model created from the apo MtMetX and MsHTA •acetyl-CoA (PDB ID 6IOI) structures with energy minimization.

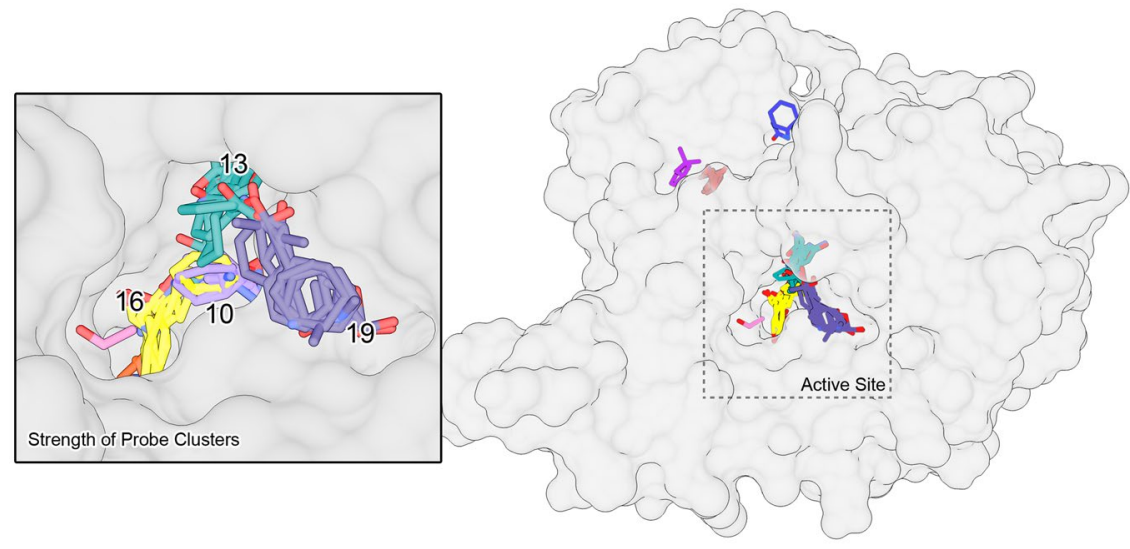

Figure 6. Probe clusters generated from the analysis of monomeric MtMetX using FTMap to evaluate druggability. A few of the critical cluster strengths, the number of probes found in each cluster, are shown in the active site inset.

In addition to sharing the overall domain and fold arrangement, the conserved location of the Ser-His-Asp catalytic triad's position within the active site tunnel may help protect the enzyme from covalent modification and deactivation. Engineered $\beta$-lactones with hydrophobic tails have already been shown to inhibit the activity 
of $\mathrm{HiHTA}$ in vitro through the formation of adducts. There may be therapeutic value in modifying their structure to enhance specificity towards $\mathrm{Mtb}^{38}$. However, their lack of in vivo inhibition of HiHTA suggests that a more efficient method for disrupting methionine biosynthesis lies in small molecule inhibitors. These would be less prone to bacterial inactivation and less prone to the exclusion by Mtb's complex lipid cell wall. The high degree of structural similarity with previously solved homologs provides an excellent foundation for in silico compound screening and structure driven drug design methodologies.

The FTMap cluster data also provides aid towards a Fragment-Based Drug Discovery (FBDD). Previous research has shown that promising core fragments typically bind in the highest strength $\mathrm{CS}^{39}$. While the fragment molecules are too small on their own to have a useful affinity, neighboring CS probe structures can be then be linked to the core fragment to build up a high-affinity ligand. Furthermore, virtually-linked fragments could be screened in silico against existing chemical homologs using a tool such as ROCs ${ }^{40}$.

M. abscessus MetX was initially chosen for study due to its high similarity to the Mtb variant. However, the structure and CS data argue that it might make an excellent secondary target for drug development. Many compounds that inhibit MtMetX are also likely to affect MaMetX. M. abscessus is an emerging public-health threat, primarily implicated in pulmonary infections ${ }^{41}$. Cross-species gene transfer has helped to create multidrug-resistant strains, some even showing resistance to TB drugs such as rifampin ${ }^{42,43}$.

In summary, by reporting the first medically relevant $M t \mathrm{MetX}$ and $\mathrm{MaMetX}$ crystal structures, we hope that new avenues of structure-based drug design will be open for developing targeted and effective therapeutics.

\section{Experimental Procedures}

Protein expression and purification. Constructs of $M t \mathrm{MetX}, M h \mathrm{MetX}$, and $M a \mathrm{Met}$ X were prepared from polymerase chain reaction (PCR) products amplified from corresponding genomic DNA and subcloned into a pCDF-NT vector. Following primers were used for amplification:

$$
\begin{aligned}
& \text { metXmha_Nco GACACCATGGCCGAAGGCGAACTCG, } \\
& \text { metXmha_Hind CTGAAGCTTATGACGCCAACTCCAACGTC, } \\
& \text { metXmab_Nco GAGACCATGGCTCTACCCCAGGGCGATGAG, } \\
& \text { metXmab_Hind CTCAAGCTTACTTGGCCAGTGCGAGCG, } \\
& \text { metXmtb_BspH GAGATCATGACGCTGCCCGCCGAAG, } \\
& \text { metXmtb_Hind CACAAGCTTAATCAGCCAATCCCAGTGTCTG. }
\end{aligned}
$$

pCDF-NT is a modified pCDF-Duet 1 plasmid (Novagen) encoding $\mathrm{His}_{6}$ tag followed by a tobacco etch virus (TEV) protease cleavage site. The pCDG-NT:His ${ }_{6}-$ MetX plasmids were transformed into Escherichia coli Rosetta (DE3) competent cells (Novagen). Constructs were grown in $\mathrm{LB}$ to an $\mathrm{OD}_{600}$ of 0.6 at $37^{\circ} \mathrm{C}$ in the presence of streptomycin and chloramphenicol. Cultures were then cooled in an ice bath to $18^{\circ} \mathrm{C}$ before the addition of $200 \mu \mathrm{M}$ Isopropyl $\beta$-D-thiogalactopyranoside (IPTG) and $2 \% \mathrm{v} / \mathrm{v}$ ethanol. After inducing overnight for 16 hours, cultures were centrifuged at 5,000 rpm and lysed through two passes through a Microfluidizer (Microfluidics). Cell debris was removed via centrifugation at $18,000 \mathrm{rpm}$ for 1 hour at $4^{\circ} \mathrm{C}$. Protein was purified by passage over a Ni-affinity column containing His-Trap chelating resin from GE Healthcare Life Sciences. The column was washed with a buffer containing $20 \mathrm{mM}$ Tris $\mathrm{pH} 8.0,300 \mathrm{mM} \mathrm{NaCl}$, and $10 \mathrm{mM}$ imidazole. The protein was then eluted using the same buffer with $250 \mathrm{mM}$ imidazole. The elution fraction was dialyzed overnight at $4{ }^{\circ} \mathrm{C}$ into $20 \mathrm{mM}$ Tris $\mathrm{pH} 8.0,150 \mathrm{mM} \mathrm{NaCl}$ alongside TEV protease to release the $\mathrm{His}_{6}$ tag. The passage of the protein back over the same His-Trap column removed the TEV protease, and tag before a polishing pass was performed over a Superdex 200 gel filtration column (GE Healthcare Life Sciences) in $20 \mathrm{mM}$ Tris 7.5, $100 \mathrm{mM}$ NaCl buffer.

Crystallization of MtMetX, MhMetX, and MaMetX. Initial crystallization screens were performed using the MCSG (Anatrace) and JCSG (Qiagen) crystallization suites on a Mosquito (TTP Labtech) in vapor-diffusion hanging-drop 96-well format plates. Each drop was created from $1 \mu \mathrm{L}$ of protein solution and $1 \mu \mathrm{L}$ well solution. Plates were allowed to grow at $18^{\circ} \mathrm{C}$ and monitored daily. Initial MhMetX crystals were obtained in $0.2 \mathrm{M} \mathrm{Ca}$ acetate, 20\% PEG3350. These crystals had spherulite-like morphology. Using Additive Screen (Hampton Research), the optimized prism-like crystals were obtained in $0.15 \mathrm{M} \mathrm{Ca}$ acetate, 21\% PEG3350, 3\% 1,6-diaminohexane, $0.05 \mathrm{M}$ CHES $\mathrm{pH}$ 9.5. Initial MaMetX crystals were obtained in $1.2 \mathrm{M} \mathrm{NaH}_{2} \mathrm{PO}_{4}, 0.8 \mathrm{M} \mathrm{K}_{2} \mathrm{HPO}_{4}, 0.2 \mathrm{M} \mathrm{Li}$ sulphate, $0.1 \mathrm{M}$ CAPS $\mathrm{pH}$ 10.5. A pH grid optimization led to final crystallization solution: $1.2 \mathrm{M} \mathrm{NaH}_{2} \mathrm{PO}_{4}, 0.8 \mathrm{M} \mathrm{K}_{2} \mathrm{HPO}_{4}$, $0.2 \mathrm{M}$ Li sulphate, $0.1 \mathrm{M}$ CHES pH 9.0.The MtMetX crystal was harvested directly from the MCSG screen with crystallization solution $0.1 \mathrm{M}$ Tris- $\mathrm{HCl}, \mathrm{pH} 8.5,1.8 \mathrm{M}$ magnesium sulfate. Each tray was immediately set up after concentrating fractions from the Superdex 200 to $13.8 \mathrm{mg} / \mathrm{mL}$. The crystal from which the data set was derived grew within 48 hours.

Data and structural determination. Crystals of each MetX construct were flash-frozen in liquid nitrogen after cryoprotection by transfer into the corresponding crystallization solutions supplemented with $20-25 \%$ glycerol. Data from $M h$ MetX and MaMetX crystals were collected at the Stanford Synchrotron Radiation Lightsource beamline 9-2 using a Dectris Pilatus 6 M detector at $1.000 \AA$ wavelength. Data from $M t$ MetX crystal were collected at the SER-CAT beamline (22-ID) at the Advanced Photon Source using an Eiger $16 \mathrm{M}$ detector at $1.000 \AA$ wavelength. Data were indexed, integrated, and scaled using XDS and XSCALE $E^{44}$.

The structure of $M h \mathrm{MetX}$ was determined by molecular replacement using Phaser ${ }^{45}$ and the structure of homoserine $O$-acetyltransferase from Bacillus anthracis (PDB ID 3I1I) as a search model. The structure of $\mathrm{MaMetX}$ was determined by molecular replacement using the MhMetX structure as a search model. The crystal structure of $M t$ MetX was determined using molecular replacement in Phaser $^{45}$ using the MaMetX structure as a 


\begin{tabular}{|c|c|c|c|}
\hline & MhMetX ${ }^{\mathrm{a}}$ PDB ID 5W8O & MaMetX PDB ID 5W8P & MtMetX PDB ID 6PUX \\
\hline \multicolumn{4}{|l|}{ Data collection } \\
\hline Space group & $P 2_{1}$ & $P 6_{5}$ & $P 2_{1} 2_{1} 2_{1}$ \\
\hline \multicolumn{4}{|l|}{ Cell dimensions: } \\
\hline$a, b, c(\AA)$ & $47.75,93.23,81.59$ & $197.92,197.92,51.30$ & $47.46,86.68,208.77$ \\
\hline$\alpha, \beta, \gamma\left(^{\circ}\right)$ & $90,94.78,90$ & $90,90,120$ & $90,90,90$ \\
\hline Resolution $(\AA)$ & $47.58-1.47(1.51-1.47)^{\mathrm{b}}$ & $49.48-1.69(1.73-1.69)$ & $44.71-1.90(1.95-1.90)$ \\
\hline $\mathrm{R}_{\text {sym }}$ & $0.040(1.013)$ & $0.124(0.963)$ & $0.143(1.361)$ \\
\hline $\mathrm{CC}_{1 / 2}{ }^{\mathrm{c}}$ & $1.0(0.695)$ & $0.994(0.678)$ & $0.997(0.672)$ \\
\hline$I / \sigma(I)$ & $18.01(1.46)$ & $8.70(1.78)$ & $11.21(1.29)$ \\
\hline Completeness (\%) & $98.3(99.1)$ & $99.3(98.9)$ & $99.8(99.7)$ \\
\hline Redundancy & $3.8(3.8)$ & $5.6(5.8)$ & $7.4(7.3)$ \\
\hline \multicolumn{4}{|l|}{ Refinement } \\
\hline Resolution $(\AA)$ & $47.58-1.47$ & $49.48-1.69$ & $44.71-1.90$ \\
\hline No. reflections (total/free) & $118,621 / 5,840$ & $128,098 / 6,439$ & $68,843 / 3,463$ \\
\hline $\mathrm{R}_{\text {work }} / \mathrm{R}_{\text {free }}$ & $0.153 / 0.183$ & $0.158 / 0.174$ & $0.168 / 0.202$ \\
\hline \multicolumn{4}{|l|}{ No. atoms: } \\
\hline Protein & 5,156 & 5,488 & 5,390 \\
\hline Ligand/ion & 12 & 44 & 60 \\
\hline Water & 593 & 787 & 514 \\
\hline \multicolumn{4}{|l|}{$B$-factors: } \\
\hline Protein & 25.2 & 20.9 & 32.4 \\
\hline Ligand/ion & 36.3 & 48.2 & 69.0 \\
\hline Water & 36.7 & 34.8 & 39.0 \\
\hline All atoms & 26.4 & 22.8 & 33.3 \\
\hline Wilson $B$ & 19.6 & 18.9 & 31.3 \\
\hline \multicolumn{4}{|l|}{ R.m.s. deviations: } \\
\hline Bond lengths $(\AA)$ & 0.005 & 0.006 & 0.003 \\
\hline Bond angles $\left({ }^{\circ}\right)$ & 0.765 & 0.789 & 0.572 \\
\hline \multicolumn{4}{|c|}{ Ramachandran distribution (\%): } \\
\hline Favored & 98.37 & 97.96 & 97.66 \\
\hline Allowed & 1.63 & 2.04 & 2.34 \\
\hline Outliers & 0 & 0 & 0 \\
\hline
\end{tabular}

Table 2. Data collection and refinement statistics. ${ }^{a}$ All data were collected from single crystals. ${ }^{b}$ Values in parentheses are for the highest-resolution shell. ${ }^{c} \mathrm{CC}_{1 / 2}$ correlation coefficient between intensities from two random half-data sets.

search model. Model building was performed using $\operatorname{Coot}^{46}$; iterative refinement was done via phenix.refine ${ }^{47,48}$. Data and refinement statistics are summarized in Table 2.

Differential scanning fluorimetry (DSF). The assay was carried out using in a range of buffers using $50 \mathrm{mM}$ Tris- $\mathrm{HCl} \mathrm{pH}$ 6.9-8.5 in 100-300 mM NaCl with $0.2 \mathrm{mg} / \mathrm{mL}$ of $M t$ MetX final concentration. $20 \mu \mathrm{L}$ of protein was loaded with a $2 x$ final concentration of SYPRO Orange dye (Thermo Fisher) and run on a CFX96 Touch qPCR system (Bio-Rad). A linear thermal ramp of $1{ }^{\circ} \mathrm{C} / \mathrm{min} ; 20^{\circ} \mathrm{C}-90^{\circ} \mathrm{C}$ run with an excitation wavelength of $512-535 \mathrm{~nm}$ and a detection wavelength of $560-580 \mathrm{~nm}$. $\mathrm{T}_{\mathrm{m}}$ was calculated as the minimum of the first derivative plot of the unfolding transition in the CFX Maestro software.

Structural comparison and fragment-based hot spot detection. The Flexible structure alignment by chaining aligned fragment pairs allowing twists (FATCAT) server (http://fatcat.burnham.org) was used to compare the different available HTA structures. All pairwise alignments were done using the flexible alignment model with chain A. The database search for close homologs was performed with a $P$-value of 0.05 . The FTMap server (http://ftmap.bu.edu) was used to map chain A of the MtMetX structure and assay ligandability. Cluster strength was determined by the number of probes in each consensus sites. The same run was also performed on the FTFlex server (https://ftlex.bu.edu) to assay whether or not sidechain flexibility would significantly alter the results, but observed trends in cluster locations were not significantly different between both servers.

The $M t$ MetX substrate models were created by using a $M s$ HTA structures, which have been solved in the presence of acetyl-CoA (PDB entries $6 \mathrm{IOH}$ and $6 \mathrm{IOI})^{24}$. Monomers from each structure positioned using rigid-body alignment using Chimera's MatchMaker ${ }^{49}$. Energy minimization on the hybrid structure was then performed using the Molecular Modeling Toolkit and Dock Prep with the AMBER ff14SB forcefield ${ }^{50}$. 


\section{Data availability}

Atomic coordinates and structure factors of the reported crystal structures have been deposited to the Protein

Data Bank with accession codes 5W8O (MhMetX), 5W8P (MaMetX), and 6PUX (MtMetX).

Received: 16 October 2019; Accepted: 16 December 2019;

Published online: 30 December 2019

\section{References}

1. Glaziou, P., Sismanidis, C., Floyd, K. \& Raviglione, M. Global epidemiology of tuberculosis. Cold Spring Harb. Perspect. Med. 5, a017798 (2014)

2. World Health Organization. Global Tuberculosis Report 2018. (World Health Organization, Geneva, Switzerland, 2018).

3. Miller, T. L., McNabb, S. J., Hilsenrath, P., Pasipanodya, J. \& Weis, S. E. Personal and societal health quality lost to tuberculosis. PLoS One 4, e5080 (2009).

4. Fletcher, H. A., Hawkridge, T. \& McShane, H. A New Vaccine for Tuberculosis: The Challenges of Development and Deployment. J. Bioeth. Inq. 6, 219-228 (2009)

5. Marks, S. M. et al. Treatment practices, outcomes, and costs of multidrug-resistant and extensively drug-resistant tuberculosis, United States, 2005-2007. Emerg. Infect. Dis. 20, 812-821 (2014).

6. Viola, R. E. et al. The catalytic machinery of a key enzyme in amino Acid biosynthesis. J. Amino Acids 2011, 352538 (2011).

7. Bastard, K. et al. Parallel evolution of non-homologous isofunctional enzymes in methionine biosynthesis. Nat. Chem. Biol. 13, 858-866 (2017)

8. Nazi, I. et al. Role of homoserine transacetylase as a new target for antifungal agents. Antimicrob. Agents Chemother. 51, 1731-1736 (2007).

9. Pascon, R. C., Ganous, T. M., Kingsbury, J. M., Cox, G. M. \& McCusker, J. H. Cryptococcus neoformans methionine synthase: expression analysis and requirement for virulence. Microbiology 150, 3013-3023 (2004).

10. Berney, M. et al. Essential roles of methionine and S-adenosylmethionine in the autarkic lifestyle of Mycobacterium tuberculosis. Proc. Natl Acad. Sci. USA 112, 10008-10013 (2015).

11. Hasenoehrl, E. J. et al. Derailing the aspartate pathway of Mycobacterium tuberculosis to eradicate persistent infection. Nat. Commun. 10, 4215 (2019).

12. Pavelka, M. S. Jr. One of these is not like the others. Trends Microbiol. 23, 668-670 (2015).

13. Pavlovsky, A. G., Liu, X., Faehnle, C. R., Potente, N. \& Viola, R. E. Structural characterization of inhibitors with selectivity against members of a homologous enzyme family. Chem. Biol. Drug. Des. 79, 128-136 (2012).

14. Nardini, M. \& Dijkstra, B. W. Alpha/beta hydrolase fold enzymes: the family keeps growing. Curr. Opin. Struct. Biol. 9, 732-737 (1999).

15. Carr, P. D. \& Ollis, D. L. Alpha/beta hydrolase fold: an update. Protein Pept. Lett. 16, 1137-1148 (2009).

16. Lenfant, N. et al. ESTHER, the database of the alpha/beta-hydrolase fold superfamily of proteins: tools to explore diversity of functions. Nucleic Acids Res. 41, D423-429 (2013).

17. Heikinheimo, P., Goldman, A., Jeffries, C. \& Ollis, D. L. Of barn owls and bankers: a lush variety of alpha/beta hydrolases. Structure 7, R141-146 (1999).

18. Krissinel, E. \& Henrick, K. Inference of macromolecular assemblies from crystalline state. J. Mol. Biol. 372, 774-797 (2007).

19. Born, T. L. \& Blanchard, J. S. Enzyme-catalyzed acylation of homoserine: mechanistic characterization of the Escherichia coli metAencoded homoserine transsuccinylase. Biochemistry 38, 14416-14423 (1999).

20. Zubieta, C., Arkus, K. A., Cahoon, R. E. \& Jez, J. M. A single amino acid change is responsible for evolution of acyltransferase specificity in bacterial methionine biosynthesis. J. Biol. Chem. 283, 7561-7567 (2008).

21. Nazi, I. \& Wright, G. D. Catalytic mechanism of fungal homoserine transacetylase. Biochemistry 44, 13560-13566 (2005).

22. Wang, M. et al. Crystal structure of homoserine O-acetyltransferase from Leptospira interrogans. Biochem. Biophys. Res. Commun. 363, 1050-1056 (2007).

23. Thangavelu, B., Pavlovsky, A. G. \& Viola, R. Structure of homoserine O-acetyltransferase from Staphylococcus aureus: the first Gram-positive ortholog structure. Acta Crystallogr. F. Struct. Biol. Commun. 70, 1340-1345 (2014).

24. Sagong, H. Y., Hong, J. \& Kim, K. J. Crystal structure and biochemical characterization of O-acetylhomoserine acetyltransferase from Mycobacterium smegmatis ATCC 19420. Biochem. Biophys. Res. Commun. 517, 399-406 (2019).

25. Mirza, I. A., Nazi, I., Korczynska, M., Wright, G. D. \& Berghuis, A. M. Crystal structure of homoserine transacetylase from Haemophilus influenzae reveals a new family of alpha/beta-hydrolases. Biochemistry 44, 15768-15773 (2005).

26. Touw, W. G. et al. A series of PDB-related databanks for everyday needs. Nucleic Acids Res 43, D364-368, (2015).

27. Kabsch, W. \& Sander, C. Dictionary of protein secondary structure: pattern recognition of hydrogen-bonded and geometrical features. Biopolymers 22, 2577-2637 (1983).

28. Hopkins, A. L. \& Groom, C. R. The druggable genome. Nat. Rev. Drug. Discov. 1, 727-730 (2002).

29. Russ, A. P. \& Lampel, S. The druggable genome: an update. Drug. Discov. Today 10, 1607-1610 (2005).

30. Vukovic, S. \& Huggins, D. J. Quantitative metrics for drug-target ligandability. Drug. Discov. Today 23, 1258-1266 (2018).

31. Sakharkar, M. K., Sakharkar, K. R. \& Pervaiz, S. Druggability of human disease genes. Int. J. Biochem. Cell Biol. 39, 1156-1164 (2007).

32. Sakharkar, M. K. \& Sakharkar, K. R. Targetability of human disease genes. Curr. Drug. Discov. Technol. 4, 48-58 (2007).

33. Fauman, E. B., Rai, B. K. \& Huang, E. S. Structure-based druggability assessment-identifying suitable targets for small molecule therapeutics. Curr. Opin. Chem. Biol. 15, 463-468 (2011).

34. Ehrt, C., Brinkjost, T. \& Koch, O. Binding site characterization - similarity, promiscuity, and druggability. Medchemcomm 10, $1145-1159$ (2019).

35. Brenke, R. et al. Fragment-based identification of druggable 'hot spots' of proteins using Fourier domain correlation techniques. Bioinformatics 25, 621-627 (2009).

36. Kozakov, D. et al. Structural conservation of druggable hot spots in protein-protein interfaces. Proc. Natl Acad. Sci. USA 108, 13528-13533 (2011).

37. Kozakov, D. et al. The FTMap family of web servers for determining and characterizing ligand-binding hot spots of proteins. Nat. Protoc. 10, 733-755 (2015).

38. De Pascale, G., Nazi, I., Harrison, P. H. \& Wright, G. D. beta-Lactone natural products and derivatives inactivate homoserine transacetylase, a target for antimicrobial agents. J. Antibiot. (Tokyo) 64, 483-487 (2011).

39. Hall, D. R., Ngan, C. H., Zerbe, B. S., Kozakov, D. \& Vajda, S. Hot spot analysis for driving the development of hits into leads in fragment-based drug discovery. J. Chem. Inf. Model. 52, 199-209 (2012).

40. Hawkins, P. C., Skillman, A. G. \& Nicholls, A. Comparison of shape-matching and docking as virtual screening tools. J. Med. Chem. 50, 74-82 (2007).

41. Mougari, F. et al. Infections caused by Mycobacterium abscessus: epidemiology, diagnostic tools and treatment. Expert. Rev. Anti Infect. Ther. 14, 1139-1154 (2016).

42. Aziz, D. B. et al. Rifabutin Is Active against Mycobacterium abscessus Complex. Antimicrob Agents Chemother 61, e00155 (2017). 
43. Medjahed, H., Gaillard, J. L. \& Reyrat, J. M. Mycobacterium abscessus: a new player in the mycobacterial field. Trends Microbiol. 18, $117-123(2010)$

44. Kabsch, W. Xds. Acta Crystallogr. D. Biol. Crystallogr 66, 125-132 (2010).

45. McCoy, A. J. et al. Phaser crystallographic software. J. Appl. Crystallogr. 40, 658-674 (2007).

46. Emsley, P. \& Cowtan, K. Coot: model-building tools for molecular graphics. Acta Crystallogr. D. Biol. Crystallogr 60, 2126-2132 (2004).

47. Afonine, P. V., Grosse-Kunstleve, R. W., Urzhumtsev, A. \& Adams, P. D. Automatic multiple-zone rigid-body refinement with a large convergence radius. J. Appl. Crystallogr. 42, 607-615 (2009).

48. Afonine, P. V. et al. Towards automated crystallographic structure refinement with phenix.refine. Acta Crystallogr. D. Biol. Crystallogr 68, 352-367 (2012).

49. Pettersen, E. F. et al. UCSF Chimera-a visualization system for exploratory research and analysis. J. Comput. Chem. 25, 1605-1612 (2004).

50. Maier, J. A. et al. ff14SB: Improving the Accuracy of Protein Side Chain and Backbone Parameters from ff99SB. J. Chem. Theory Comput. 11, 3696-3713 (2015).

\section{Acknowledgements}

The work in this study was supported by an Institutional Development Award (IDeA) from the National Institute of General Medical Sciences of the National Institutes of Health grants P20GM103486 and P30GM110787 to K.V.K. E.S.R. was supported by the National Science Foundation Research Experiences for Undergraduates (REU) grant 1358627. E.S.R. is the American Chemical Society Scholar. C.W.K. was supported by a Howard Hughes Medical Institute undergraduate science education grant to Georgetown College and the Georgetown College Science Honors Program. Use of the Stanford Synchrotron Radiation Lightsource, SLAC National Accelerator Laboratory, is supported by the U.S. Department of Energy, Office of Science, Office of Basic Energy Sciences under Contract No. DE-AC02-76SF00515. The SSRL Structural Molecular Biology Program is supported by the DOE Office of Biological and Environmental Research and by the National Institutes of Health, National Institute of General Medical Sciences (P41GM103393). Use of SER-CAT is supported by its member institutions (see www.ser-cat.org/members.html), and equipment grants (S10_RR25528 and S10_RR028976) from the National Institutes of Health. Use of the Advanced Photon Source was supported by the U. S. Department of Energy, Office of Science, Office of Basic Energy Sciences, contract W-31-109-Eng-38. The contents of this publication are solely the responsibility of the authors and do not necessarily represent the official views of NIGMS or NIH.

\section{Author contributions}

C.T.C. and K.V.K. planned the experiments. C.T.C., E.S.R., R.W.R., J.L., C.W.K., and K.V.K. performed the experiments. C.T.C. and K.V.K. analyzed the data and wrote the manuscript. All other authors reviewed the manuscript.

\section{Competing interests}

The authors declare no competing interests.

\section{Additional information}

Correspondence and requests for materials should be addressed to K.V.K.

Reprints and permissions information is available at www.nature.com/reprints.

Publisher's note Springer Nature remains neutral with regard to jurisdictional claims in published maps and institutional affiliations.

(c) (i) Open Access This article is licensed under a Creative Commons Attribution 4.0 International

License, which permits use, sharing, adaptation, distribution and reproduction in any medium or format, as long as you give appropriate credit to the original author(s) and the source, provide a link to the Creative Commons license, and indicate if changes were made. The images or other third party material in this article are included in the article's Creative Commons license, unless indicated otherwise in a credit line to the material. If material is not included in the article's Creative Commons license and your intended use is not permitted by statutory regulation or exceeds the permitted use, you will need to obtain permission directly from the copyright holder. To view a copy of this license, visit http://creativecommons.org/licenses/by/4.0/.

(c) The Author(s) 2019 